\title{
Resonant tunneling diodes made up of stacked self-assembled $\mathrm{Ge} / \mathrm{Si}$ islands
}

O. G. Schmidt, U. Denker, K. Eberl, O. Kienzle, F. Ernst, and R. J. Haug

Citation: Appl. Phys. Lett. 77, 4341 (2000); doi: 10.1063/1.1332817

View online: https://doi.org/10.1063/1.1332817

View Table of Contents: http://aip.scitation.org/toc/apl/77/26

Published by the American Institute of Physics

\section{Articles you may be interested in}

$\mathrm{Si} / \mathrm{SiGe}$ electron resonant tunneling diodes

Applied Physics Letters 77, 1653 (2000); 10.1063/1.1309020

Resonant tunneling in $\mathrm{Si} / \mathrm{Si}_{1-x} \mathrm{Ge}_{x}$ double-barrier structures

Applied Physics Letters 52, 1809 (1998); 10.1063/1.99632

Oscillations up to $712 \mathrm{GHz}$ in InAs/AISb resonant-tunneling diodes

Applied Physics Letters 58, 2291 (1991); 10.1063/1.104902

Symmetric $\mathrm{Si} / \mathrm{Si}_{1-x} \mathrm{Ge}_{\mathrm{x}}$ electron resonant tunneling diodes with an anomalous temperature behavior Applied Physics Letters 62, 603 (1993); 10.1063/1.108869

Room temperature operation of epitaxially grown $\mathrm{Si} / \mathrm{Si}_{0.5} \mathrm{Ge}_{0.5} / \mathrm{Si}$ resonant interband tunneling diodes Applied Physics Letters 73, 2191 (1998); 10.1063/1.122419

Band lineup of layered semiconductor heterointerfaces prepared by van der Waals epitaxy: Charge transfer correction term for the electron affinity rule

Journal of Applied Physics 85, 2732 (1999); 10.1063/1.369590

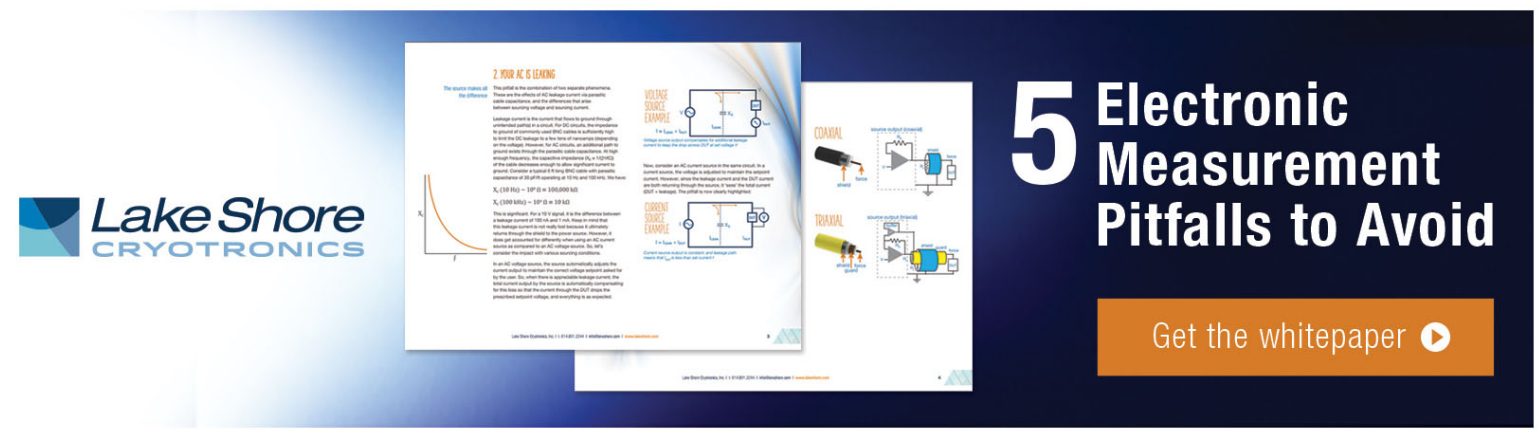




\title{
Resonant tunneling diodes made up of stacked self-assembled $\mathrm{Ge} / \mathrm{Si}$ islands
}

\author{
O. G. Schmidt, ${ }^{\text {a) }}$ U. Denker, and K. Eberl \\ Max-Planck-Institut für Festkörperforschung, Heisenbergstrasse 1, 70569 Stuttgart, Germany \\ O. Kienzle and F. Ernst \\ Max-Planck-Institut für Metallforschung, Seestrasse 92, 70174 Stuttgart, Germany \\ R. J. Haug \\ Institut für Festkörperphysik, Universität Hannover, Appelstrasse 2, 30167 Hannover, Germany
}

(Received 27 July 2000; accepted for publication 23 October 2000)

\begin{abstract}
Multiple layers of self-assembled $\mathrm{Ge} / \mathrm{Si}$ islands are used for resonant tunneling diodes (RTDs). The extremely closely stacked Ge nanostructures form vertical channels with energetically deep thermalization layers and high Si double barriers. Two resonances are found in the RTD currentvoltage curve, which are attributed to the heavy-heavy hole (hh) and heavy-light hole (lh) transition. The lh resonance shows negative differential resistance up to $50 \mathrm{~K}$. With increasing magnetic field, the lh resonance slightly shifts to higher voltages. (C) 2000 American Institute of Physics.
\end{abstract}

[S0003-6951(00)01951-3]

Self-assembled $\mathrm{Ge} / \mathrm{Si}$ islands have been of broad interest during the last years and a lot of work has been put into their structural, ${ }^{1-4}$ vibrational, ${ }^{5}$ optical, ${ }^{4,6-9}$ and electronic investigation. ${ }^{10}$ Despite the large effort to understand their fundamental properties, devices incorporating $\mathrm{Ge} / \mathrm{Si}$ islands as active material are only scarcely found. Resonant tunneling diodes (RTDs) with pseudomorphic $\mathrm{SiGe} / \mathrm{Si}$ quantum wells on $\mathrm{Si}$ substrates are commonly described in the literature. ${ }^{11-13}$ However, their preformance is rather poor, yielding negative differential resistance (NDR) only up to temperatures of about $77 \mathrm{~K}$. Valence band (VB) offsets of typical RTDs are about $200 \mathrm{meV}$ (Ge concentrations of 20\%) and thermal quenching plays a major role at higher temperatures. In this letter we introduce a new concept for resonant tunneling diodes, which makes use of self-assembled Ge islands. First results of an island based RTD are presented. The purpose of the concept is twofold: First, the large valence band offsets of $\mathrm{Ge}$ islands are used to create deep Ge rich vertical channels with high Si double barriers. Second, vertically self-aligned $\mathrm{Ge} / \mathrm{Si}$ island stacks offer the possibility of perfect side passivation, since they are coherently embedded in the $\mathrm{Si}$ matrix.

Figure 1(a) shows a cross-sectional transmission electron microscopy (TEM) image of a typical active layer arrangement in quantum well RTDs. Two thick $\mathrm{Si}_{1-x} \mathrm{Ge}_{x}$ quantum wells at the bottom and the top serve as thermalization layers for holes. The actual RTD is created by a thin $\mathrm{Si}_{1-x} \mathrm{Ge}_{x}$ quantum well, sandwiched between two Si barriers. The schematic band edge alignment for the VB is given next to the TEM image.

A different concept is presented in Fig. 1(b). It is based on the vertical self-alignment of closely stacked $\mathrm{Ge} / \mathrm{Si}$ islands. ${ }^{14-18}$ In this case the thermalization layers are created by very closely stacked $\mathrm{Ge}$ islands yielding small minibands due to strong electronic carrier coupling. The two Si barriers

${ }^{a)}$ Electronic mail: oschmidt@ servix.mpi-stuttgart.mpg.de are formed by leaving a slightly thicker spacer layer between the fifth and sixth as well as the sixth and seventh island layer. The schematic band edge alignment (without bias and doping) yields much larger valence band offsets than for the quantum well case. A slight modification of this concept can be found in Fig. 1(c), where the first two island layers are substituted by a thick SiGe quantum well.

We concentrate our tunneling experiments on one sample which was grown by solid source molecular beam epitaxy. The exact growth procedure follows the concept of Fig. 1(c)-with five instead of nine island layers: On a $p^{+} \mathrm{Si}(001)$ substrate a $100 \mathrm{~nm} \mathrm{Si:B}\left(1 \times 10^{19} \mathrm{~cm}^{-2}\right)$ layer is grown followed by a $12 \mathrm{~nm}$ thick $\mathrm{Si}_{0.82} \mathrm{Ge}_{0.12}$ quantum well. After that a stack of five bilayers of $6 \mathrm{ML} \mathrm{Ge}$ and Si layers is deposited, where the Si spacer layer between the first and second and second and third island layer is $9 \mathrm{~nm}$ and the other $\mathrm{Si}$ spacers are only $5 \mathrm{~nm}$ thick. The structure is finished with a $150 \mathrm{~nm}$ thick Si:B $\left(1 \times 10^{19} \mathrm{~cm}^{-2}\right)$ cap. The growth temperature for the $\mathrm{Ge}$ islands was $600^{\circ} \mathrm{C}$, resulting in low density and large Ge islands with diameters of about $80 \mathrm{~nm}^{4}$ Single embedded layers of such islands emit at $0.77 \mathrm{eV}$. Neglecting strain induced electron localization this yields a VB offset of about $400 \mathrm{meV}$. The sample is processed into mesas. We use optical lithography and wet-chemical etching with $\mathrm{HNO}_{3}$ :HF. A typical pillar is given in Fig. 2(a). Pronounced underetching is evident. Small dips in the sidewall indicate different etching rates for the $\mathrm{Si}$ and $\mathrm{SiGe}$ epilayers. The presented mesa is $400 \mathrm{~nm}$ wide. For such diameters we expect only a few island stacks in the mesa. Large area Ti/Au contacts are then evaporated onto a polymide passivated surface [see Fig. 2(b)]. Electrical measurements are carried out in a ${ }^{3} \mathrm{He}$ evaporation cryostat equipped with a $12 \mathrm{~T}$ magnet. The direction of the current flow through the RTD is indicated in the inset of Fig. 3.

The $I-V$ curve of the RTD for positive bias is presented in Fig. 3 (solid curve) and shows two resonances, one at 0.49 $\mathrm{V}$, which exhibits negative differential resistance and a sec- 

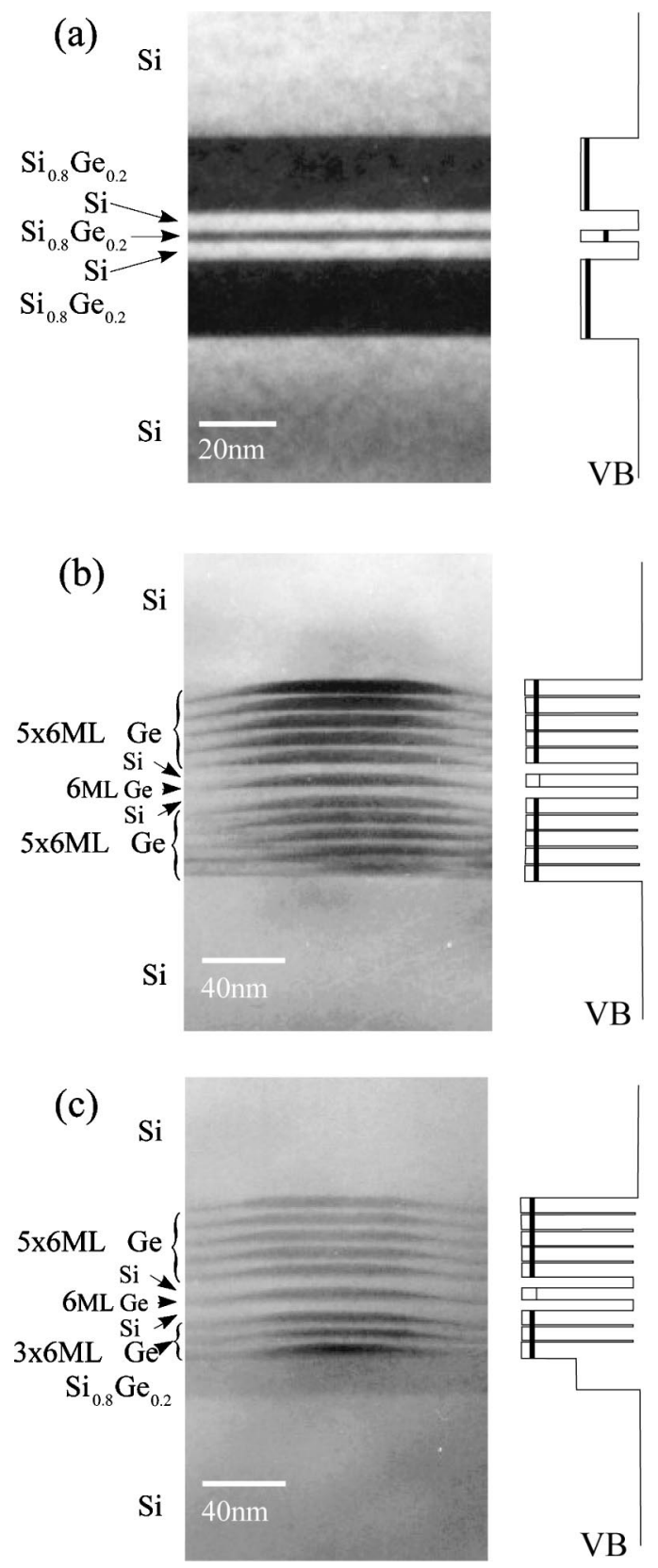

FIG. 1. Cross-section TEM images illustrating three different approaches to realize resonant tunneling diodes based on the SiGe material system. Schematic band alignment without doping and bias is shown next to the TEM images. (a) Shows the conventional quantum well case, whereas in (b) and (c) Ge islands are used for the thermalization layers and the resonant tunneling structure.

ond shoulder at about $0.34 \mathrm{~V}$. The two features can be explained by resonant tunneling of hhs through the hh and $\mathrm{lh}$ subbands of the middle Ge island. The $I-V$ characteristic agrees with those observed for conventional RTDs using $\mathrm{Si} /$
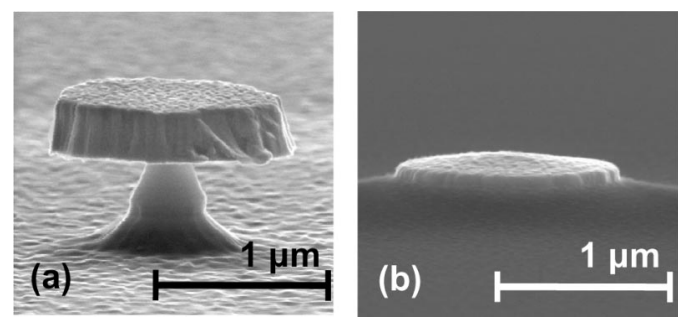

FIG. 2. (a) Wet-chemically etched mesa, (b) buried by polymid.

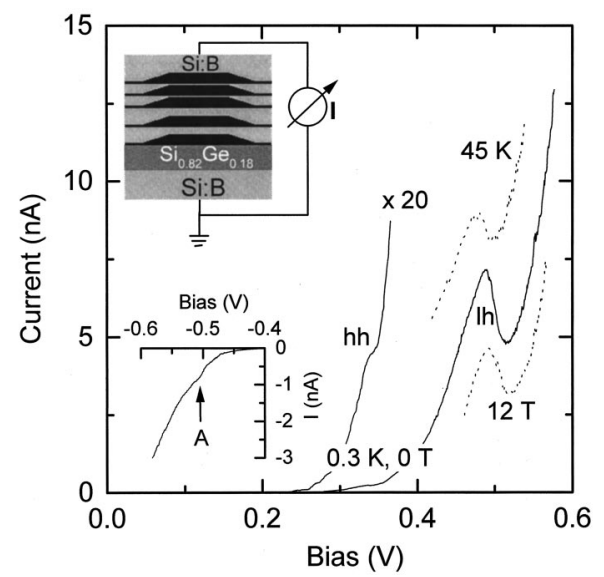

FIG. 3. Three $I-V$ curves of the RTD for different temperatures and magnetic fields. The solid line shows the measurement at $0.3 \mathrm{~K}$ without magnetic field. The lower dotted curve is taken at $0.3 \mathrm{~K}$ and $12 \mathrm{~T}$ and the upper dotted curve was recorded at $45 \mathrm{~K}$ without magnetic field. The lower left inset shows the $I-V$ characteristic for negative bias $(0.3 \mathrm{~K}$ without magnetic field). The upper left inset is a schematic illustration of the RTD.

SiGe quantum wells. ${ }^{12}$ Figure 3 also shows (upper dotted curve) that NDR persists for temperatures larger than $45 \mathrm{~K}$. The peak to valley current ratio (PVCR) as a function of temperature is given in Fig. 4(a). At $0.3 \mathrm{~K}$ the PVCR is 1.5 , whereas at $60 \mathrm{~K}$ only a shoulder is resolved in the $I-V$ characteristic.

The $I-V$ curve around the hh-lh resonance for a magnetic field $B=12 \mathrm{~T}$ is given in Fig. 3 (lower dotted curve). The magnetic field is applied parallel to the current. There is a slight shift to higher voltages with increasing $B$ as illustrated in Fig. 4(b). The magnetic field constrains the electronic states into Landau levels, which split as $\Delta E$ $\sim B / m_{\text {eff }}$, where $m_{\text {eff }}$ is the effective mass. Since the effective masses are different in the emitter (heavy holes) and in the middle island (light holes) the whole resonance is expected to shift linearly with increasing $B .{ }^{12}$ We find a shift of $12 \mathrm{mV}$ for $B=12 \mathrm{~T}$, which is similar to values reported for quantum well based RTDs. In Ref. 12 for example a shift of $10 \mathrm{mV}$ at $12 \mathrm{~T}$ was found. A slightly nonlinear dependence
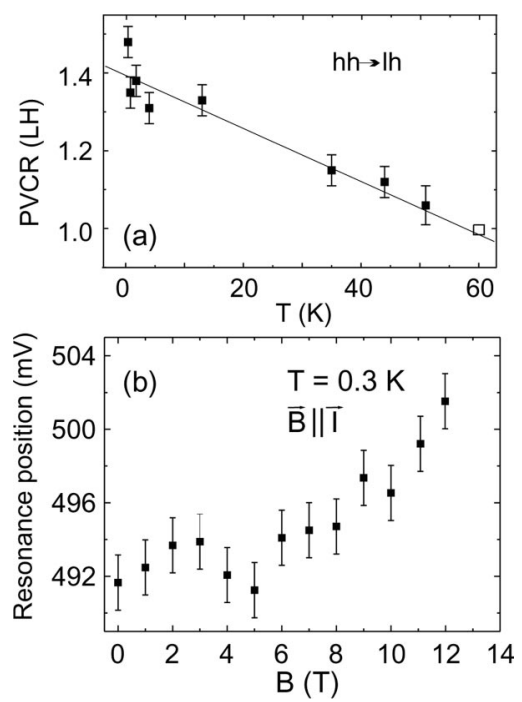

FIG. 4. (a) Peak to valley current ratio (PVCR) as a function of temperature. (b) Resonance position of the $\mathrm{hh}-\mathrm{lh}$ resonance as a function of magnetic field applied parallel to the current. 
for magnetic fields smaller than $5 \mathrm{~T}$ is observed in our structure as well as in the QW RTD in Ref. 12. The similarity between our island and the QW RTDs suggests a twodimensional density of states in the Ge islands. This is a reasonable assumption since the lateral extension of the islands is much larger than the de Broglie wavelength of charge carriers in the semiconductor.

It is noteworthy that our $I-V$ curve is not symmetric around zero bias and only shows a very weak resonance at $-0.5 \mathrm{~V}$ (marked as $\mathrm{A}$ in the lower-left inset of Fig. 3). We attribute this asymmetry to the pronounced nonsymmetric shape and alloy profile of the Ge islands (see Fig. 1). This leads to differently thick Si barriers on the emitter and collector side and destroys the symmetric behavior of the resonances in the $I-V$ curve.

Although our first results look very promising we point out some problems faced during the fabrication of such RTDs: An ideal RTD would be based on a single island stack. For postepitaxial processing the position of this specific island stack must be precisely known. Consequently, controlled lateral ordering of self-assembling islands is an important prerequisite for further improvement of the RTD's $I-V$ characteristic. Recently, we showed that the degree of materials intermixing in upper island layers is more pronounced.9,19 Additionally, penetrating strain fields and different shapes and sizes of buried islands ${ }^{16-18}$ are likely to modify the electronic band structure in the island stacks and in the sandwiched Si barriers. These effects make it difficult to produce vertically homogeneous islands and hence to evaluate the exact band edge alignment. On the other hand, it is well known from QW RTDs that a careful tuning of the band edge alignment is essential to obtain good device performance. Hence, the fundamental electronic properties of $\mathrm{Ge} / \mathrm{Si}$ island stacks need to be investigated further to improve the RTD results presented in this letter.

In conclusion, we have introduced a concept to fabricate RTDs based on stacked Ge/Si islands. The concept exploits the effect of vertical self-alignment and creates vertical Ge channels with larger valence band offsets than in conventional $\mathrm{Si} / \mathrm{SiGe}$ quantum well RTDs. Our RTD sample shows pronounced resonance peaks in the $I-V$ curve, which are attributed to the $\mathrm{hh}$ and $\mathrm{lh}$ resonant tunneling currents. The $\mathrm{lh}$ resonance is distinct and exhibits negative differential resistance for temperatures up to $50 \mathrm{~K}$. Magnetic field dependent measurements suggest a 2D density of states in the Ge islands. Problems in the fabrication of homogeneous islands are addressed.

The authors thank C. Lange for the SEM images. This work was supported by the Bundesministerium für Bildung und Forschung (Project No. 01M 2953 A0).

${ }^{1}$ G. Medeiros-Ribeiro, A. M. Bratkovski, T. I. Kamins, D. A. A. Ohlberg, and R. S. Williams, Science 279, 353 (1998), and references therein.

${ }^{2}$ S. Christiansen, M. Albrecht, and H. P. Strunk, Appl. Phys. Lett. 64, 3617 (1994)

${ }^{3}$ M. Goryll, L. Vescan, K. Schmidt, S. Mesters, H. Lüth, and K. Szot, Appl. Phys. Lett. 71, 410 (1997).

${ }^{4}$ O. G. Schmidt, C. Lange, and K. Eberl, Appl. Phys. Lett. 75, 1905 (1999).

${ }^{5}$ J. L. Liu, G. Jin, Y. S. Tang, Y. H. Luo, and K. L. Wang, Appl. Phys. Lett. 76, 586 (2000).

${ }^{6}$ H. Sunamura, N. Usami, Y. Shiraki, and S. Fukatsu, Appl. Phys. Lett. 66, 3024 (1995).

${ }^{7}$ L. Vescan and T. Stoica, J. Lumin. 80, 485 (1999).

${ }^{8}$ V. Le Thanh, P. Boucaud, D. Debarre, Y. Zheng, D. Bouchier, and J.-M. Lourtioz, Phys. Rev. B 58, 13115 (1998).

${ }^{9}$ O. G. Schmidt and K. Eberl, Phys. Rev. B 61, 13721 (2000).

${ }^{10}$ T. Meyer, M. Klemec, and H. von Känel, Phys. Rev. B 60, R8493 (1999).

${ }^{11}$ H. C. Liu, D. Landheer, M. Buchanan, and D. C. Houghton, Appl. Phys. Lett. 52, 1809 (1988).

${ }^{12}$ G. Schuberth, G. Abstreiter, E. Gornik, F. Schäffler, and J. F. Luy, Phys. Rev. B 43, 2280 (1991).

${ }^{13}$ A. Zaslavsky, D. A. Grützmacher, S. Y. Lin, T. P. Smith III, R. A. Kiehl, and T. O. Sedgwick, Phys. Rev. B 47, 16036 (1993).

${ }^{14}$ V. Rahmati, W. Jäger, H. Trinkaus, R. Loo, L. Vescan, and H. Lüth, Appl. Phys. A: Mater. Sci. Process. 62, 575 (1996).

${ }^{15}$ A. A. Darhuber, P. Schittenhelm, V. Hol, J. Stangl, G. Bauer, and G. Abstreiter, Phys. Rev. B 55, 15652 (1997).

${ }^{16}$ E. Mateeva, P. Sutter, J. C. Bean, and M. G. Lagally, Appl. Phys. Lett. 71, 3233 (1997).

${ }^{17}$ O. Kienzle, F. Ernst, M. Rühle, O. G. Schmidt, and K. Eberl, Appl. Phys. Lett. 74, 269 (1999).

${ }^{18}$ O. G. Schmidt, O. Kienzle, Y. Hao, K. Eberl, and F. Ernst, Appl. Phys. Lett. 74, 1272 (1999).

${ }^{19}$ O. G. Schmidt, K. Eberl, O. Kienzle, F. Ernst, S. Christiansen, and H. P. Strunk, Mater. Sci. Eng., B 74, 248 (2000). 